\title{
Rapid differentiation of Xihuangcao from the three Isodon species by UPLC-ESI-QTOF-MS/ MS and chemometrics analysis
}

\author{
Lai Lai Wong ${ }^{1}$, Zhitao Liang ${ }^{1,2}$, Hubiao Chen ${ }^{1,2}$ and Zhongzhen Zhao ${ }^{1,2^{*}}$
}

\begin{abstract}
Background: Isodon lophanthoides, I. lophanthoides var. graciliflorus and I. serra are the three botanical sources of Xihuangcao, which are often used indiscriminately in herbal products. The aim of this study was to develop a rapid and accurate analytical method to identify the three different botanical sources of Xihuangcao by combining UPLCESI-QTOF-MS with chemometrics analysis.

Methods: Fifteen batches of plants were collected as reference materials and their chemical profiles were analyzed by UPLC-ESI-QTOF-MS. These data were subsequently processed by statistical methods, including principal component analysis (PCA), hierarchical cluster analysis (HCA) and orthogonal partial least squared discriminant analysis (OPLS-DA). An automated sample class prediction model was also built using Naive Bayes as a class prediction algorithm to rapidly determine the source species of twenty-seven batches of commercial Xihuangcao samples.

Results: The base peak chromatograms of the three authenticated species showed different patterns and twentyseven peaks were chosen, including six diterpenoids, one phenolic acid and two glycosides to distinguish among these three species. The results showed good differentiation among the three species by PCA, HCA and OPLS-DA. Isodon lophanthoides var. graciliflorus was found to be the major botanical source of the commercial samples.
\end{abstract}

Conclusion: UPLC-ESI-QTOF-MS and subsequent chemometrics analysis were demonstrated effective to differentiate among the three different species of plants used as Xihuangcao.

\section{Background}

Xihuangcao is a folk medicine that is commonly used in southern China as a dampness-draining, antiicteric and liver protection herb [1]. Furthermore, clinical research has highlighted the beneficial effects of Xihuangcao for the treatment of hepatitis with jaundice, leading to the development and commercialization of numerous proprietary medicines and functional food products based on Xihuangcao [1]. Four different plants have been recorded as sources of Xihuangcao, including Isodon lophanthoides (Buch.-Ham. ex D. Don) Hara (IL), I. lophanthoides var. graciliflorus (Benth.) H. Hara (ILG), I. lophanthoides var. gerardianus (Benth.) H. Hara and I. serra (Maxim.) Kudo

\footnotetext{
*Correspondence: zzzhao@hkbu.edu.hk

1 School of Chinese Medicine, Hong Kong Baptist University, Kowloon, Hong Kong Special Administrative Region, People's Republic of China Full list of author information is available at the end of the article
}

(IS) [2]. According to a taxonomic revision, the plants in this genus have been much confused, especially for the varieties of $I$. lophanthoides. A new classification of I. lophanthoides and its varieties was suggested in 2004, in which I. lophanthoides var. gerardianus and I. lophanthoides var. graciliflorus were merged as I. lophanthoides var. graciliflorus (ILG) [3]. However, some experts believe that IS should not be used as Xihuangcao because its leaves do not have yellow juice when they are rubbed, which is recorded as a major characteristic of Xihuangcao in several ancient herbal medicine books [4]. Recent studies have also demonstrated the chemical compositions of IL, ILG and IS are different, which diterpenoids in IL and ILG are mainly abietane and tricyclic types and that of IS are ent-kaurane type; thus, they should not be used as one herb [1,4].

Although Xihuangcao has multiple botanical sources, most of the Xihuangcao-based products do not 
specify the species used. The development of an analytical method to identify the three different source species of Xihuangcao is therefore strongly desired to ensure the safe and effective use of these herbal products. IL and its variety ILG are difficult to distinguish based on their morphological or microscopic features $[5,6]$. In contrast, IS can be readily distinguished from IL and ILG based on its microscopic features, but this method cannot be applied to differentiate between samples of different sources in their extracted forms. It is noteworthy that Xihuangcao has not yet been recorded in The Pharmacopoeia of the People's Republic of China. The only official method available for the analysis of Xihuangcao is recorded in the Guangdong Chinese Materia Medica Standards. According to this method, samples for analysis are compared with a reference material (I. lophanthoides var. graciliflorus) by thin-layer chromatography with no provision for differentiating between the three species [2]. Although numerous studies have been conducted in recent years concerning Xihuangcao, most of these studies have limited to the chemical separation or pharmacological evaluation [7-10]. A few chemical analytical studies have been published, but each study analyzed a single species only $[11,12]$.

Chromatographic fingerprinting is an effective analytical method for identifying samples based on a comparison of their chemical profiles. Ultra-performance liquid chromatography (UPLC) can be used to separate a wide variety of compounds with superior resolution over a short period of time, and can also be coupled with mass spectrometry (MS). The major drawback of this method is that it can be time consuming to analyze all the MS data generated from a large number of samples. Chemometrics solves this problem by statistical analysis to distinguish between different species, thereby avoiding the manual comparison of huge amounts of raw MS data. Furthermore, class prediction models, which can be built based on the statistical interpretation of known reference samples can be used for automated classification of unknown samples.

The aim of this study was to identify the three botanical sources of Xihuangcao by ultra-performance liquid chromatography coupled with electrospray ionization quadrupole time-of-flight mass spectrometry (UPLCESI-QTOF-MS) in combination with chemometrics analysis.

\section{Methods}

\section{Chemicals and reference standards}

All chemicals and reference standards used in this study were purchased as the HPLC grade. Oridonin (purity $\geq 98 \%$ ) and rosmarinic acid (purity $\geq 98 \%$ ) were purchased from Chengdu Must Bio-Technology Co., Ltd (Chengdu, China). Schaftoside (purity $\geq 98 \%$ ) was purchased from Chengdu Biopurify Phytochemicals Ltd (Chengdu, China). Methanol and acetonitrile were purchased from E. Merck (Darmstadt, Germany). Formic acid (purity 96.0\%) was purchased from Tedia Company Inc. (Fairfield, OH, USA). Water was obtained from a Milli-Q water purification system (Millipore, Bedford, MA, USA).

\section{Plant materials and sample preparation}

Fifteen batches of plant samples were collected in southern China as reference materials, including four batches of I. lophanthoides var. lophanthoides, seven batches of I. lophanthoides var. graciliflorus and four batches of I. serra (Table 1; Fig. 1). These samples were authenticated

Table 1 Plant samples of Xihuangcao collected

\begin{tabular}{|c|c|c|c|}
\hline Sample no. & Species & Source & Date of collection \\
\hline $\mathrm{IL}-01$ & Isodon lophanthoides & Baiyun, Guangdong Province (transplant from Fujian) & July 2012 \\
\hline $\mathrm{IL}-02$ & Isodon lophanthoides & Baiyun, Guangdong Province & July 2012 \\
\hline $\mathrm{IL}-03$ & Isodon lophanthoides & Yingde, Guangdong Province & July 2012 \\
\hline $\mathrm{IL}-04$ & Isodon lophanthoides & Yingde, Guangdong Province & July 2012 \\
\hline ILG-01 & Isodon lophanthoides var. graciliflora & Pingyuan, Guangdong Province & July 2012 \\
\hline ILG-02 & Isodon lophanthoides var. graciliflora & Pingyuan, Guangdong Province & July 2012 \\
\hline ILG-03 & Isodon lophanthoides var. graciliflora & Heping, Guangdong Province & July 2012 \\
\hline ILG-04 & Isodon lophanthoides var. graciliflora & Baiyun, Guangdong Province & July 2012 \\
\hline ILG-04 & Isodon lophanthoides var. graciliflora & Shatin, Hong Kong & August 2013 \\
\hline ILG-05 & Isodon lophanthoides var. graciliflora & Meizhou, Guangdong Province & September 2013 \\
\hline ILG-06 & Isodon lophanthoides var. graciliflora & Panyu, Guangdong Province & February 2014 \\
\hline $\mid S-01$ & Isodon serra & Yingde, Guangdong Province & July 2012 \\
\hline IS-02 & Isodon serra & Baiyun, Guangdong Province & July 2012 \\
\hline IS-03 & Isodon serra & Zhaoqing, Guangdong Province & July 2012 \\
\hline IS-04 & Isodon serra & Panyu, Guangdong Province & February 2014 \\
\hline
\end{tabular}



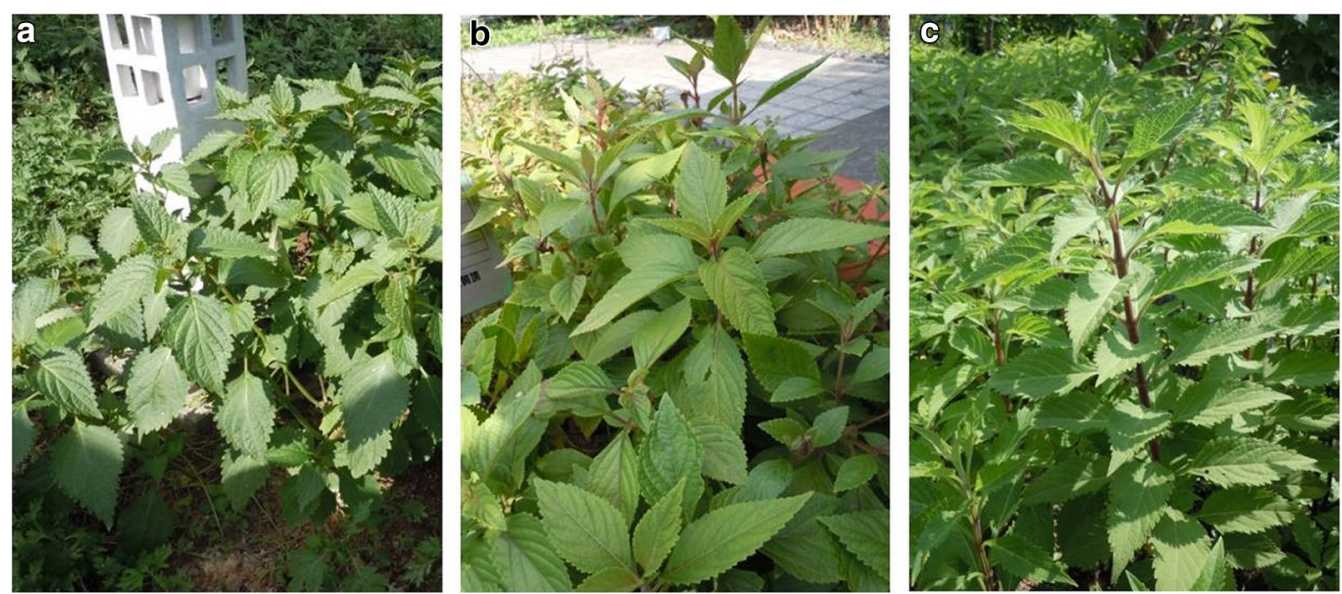

Fig. 1 The botanical sources of Xihuangcao: a I. lophanthoides, b I. lophanthoides var. graciliflorus and $\mathbf{c}$ l. serra

by Prof. Zhongzhen Zhao (School of Chinese Medicine, Hong Kong Baptist University, Hong Kong) based on their morphological characteristics [3, 13]. Twenty-seven samples of commercial batches of Xihuangcao were collected from retail markets in Guangdong and Guangxi (Table 2; Fig. 2).

Table 2 Commercial Xihuangcao samples collected and the identification result

\begin{tabular}{|c|c|c|c|}
\hline Sample no. & Producing area & Usage & Species identified \\
\hline Sample 01 & Shangsha, Guangdong & Ingredient of soup & Isodon lophanthoides var. graciliflorus \\
\hline Sample 02 & Meizhou, Guangdong & Ingredient of soup & Isodon lophanthoides var. graciliflorus \\
\hline Sample 03 & Guangxi & Herb tea & Ampelopsis grossedentata \\
\hline Sample 04 & Huizhou, Guangdong & Herb tea & Ampelopsis grossedentata \\
\hline Sample 05 & Yulin, Guangxi & Decoction pieces & Isodon serra \\
\hline Sample 06 & Yulin, Guangxi & Herb tea & Ampelopsis grossedentata \\
\hline Sample 07 & Shaoguan, Guangdong & Herb tea & Isodon serra \\
\hline Sample 08 & Meizhou, Guangdong & Ingredient of soup & Isodon lophanthoides var. graciliflorus \\
\hline Sample 09 & Taiping, Guangxi & Herb tea & Ampelopsis grossedentata \\
\hline Sample 10 & Wengyuan, Guangdong & Herb tea & Isodon serra \\
\hline Sample 11 & Wengyuan, Guangdong & Herb tea & Ampelopsis grossedentata \\
\hline Sample 12 & Guangdong & Herb tea & Isodon serra \\
\hline Sample 13 & Meizhou, Guangdong & Ingredient of soup & Isodon lophanthoides var. graciliflorus \\
\hline Sample 14 & Heyuan, Guangdong & Herb tea & Ampelopsis grossedentata \\
\hline Sample 15 & Guangdong & Herb tea & Ampelopsis grossedentata \\
\hline Sample 16 & Meizhou, Guangdong & Ingredient of soup & Isodon lophanthoides var. graciliflorus \\
\hline Sample 17 & Meizhou, Guangdong & Ingredient of soup & Unknown \\
\hline Sample 18 & Jiangxi & Decoction pieces & Isodon serra \\
\hline Sample 19 & Meizhou, Guangdong & Ingredient of soup & Isodon lophanthoides var. graciliflorus \\
\hline Sample 20 & Huizhou, Guangdong & Herb tea & Ampelopsis grossedentata \\
\hline Sample 21 & Meizhou, Guangdong & Ingredient of soup & Isodon lophanthoides var. graciliflorus \\
\hline Sample 22 & Meizhou, Guangdong & Ingredient of soup & Isodon lophanthoides var. graciliflorus \\
\hline Sample 23 & Longyan, Fujian & Herb tea & Isodon lophanthoides var. graciliflorus \\
\hline Sample 24 & Meizhou, Guangdong & Ingredient of soup & Isodon lophanthoides var. graciliflorus \\
\hline Sample 25 & Meizhou, Guangdong & Ingredient of soup & Isodon lophanthoides var. graciliflorus \\
\hline Sample 26 & Meizhou, Guangdong & Decoction pieces & Isodon serra \\
\hline Sample 27 & Meizhou, Guangdong & Decoction pieces & Isodon lophanthoides var. graciliflorus \\
\hline
\end{tabular}



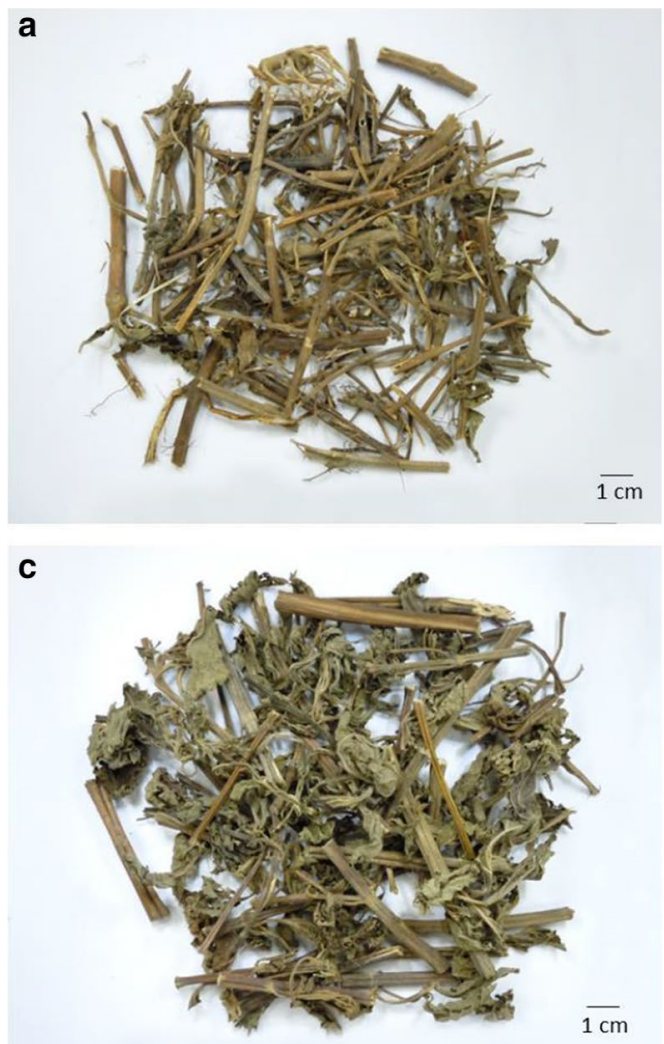

b

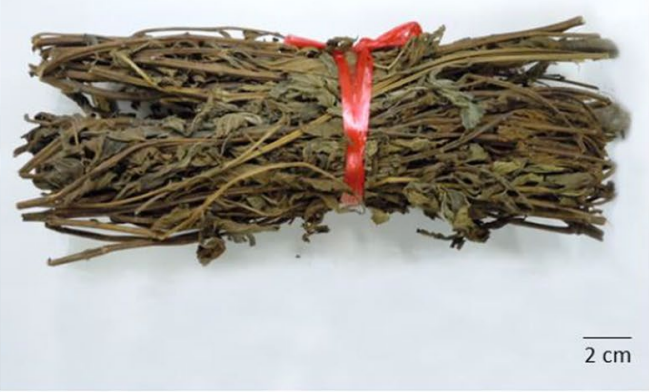

d

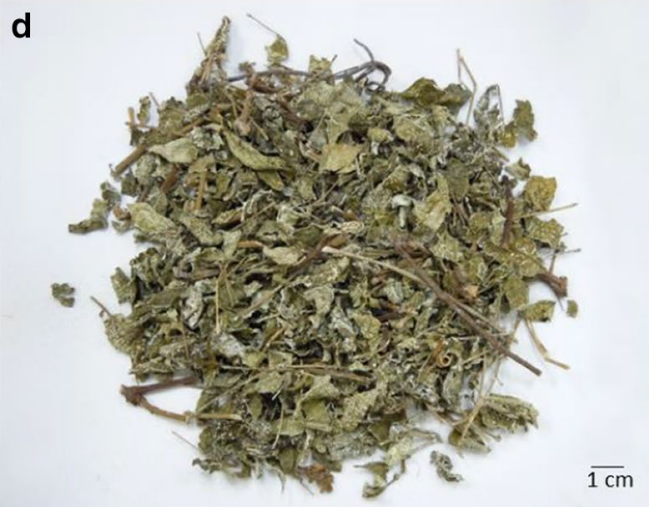

Fig. 2 Photos of some of the commercial Xihuangcao samples collected in this study: a, b I. lophanthoides var. graciliflorus, c I. serra and $\mathbf{d}$ Ampelopsis grossedentata

For the preparation of the reference materials, the roots were removed from the plants together with any foreign matter, and the remaining materials were dried under the sun. All of the dried samples were then powdered in a blender. A sample $(0.5 \mathrm{~g})$ of the powdered material was then accurately weighed into a centrifuge tube followed by $10 \mathrm{~mL}$ of methanol, and the resulting mixture was sonicated for $30 \mathrm{~min}$ at room temperature. The supernatant was then centrifuged at $25,920 \times g$ for $10 \mathrm{~min}$ on an Eppendorf 5810 centrifuge (Eppendorf, Hamburg, Germany). The extract $(0.2 \mathrm{~mL})$ was then diluted with $0.8 \mathrm{~mL}$ of methanol and transferred into a brown HPLC vial for injection.

\section{UPLC-ESI-QTOF-MS analysis}

UPLC-ESI-QTOF-MS analysis was performed on an Agilent 6540 accurate mass Q-TOF LC/MS system (Agilent Technologies, Santa Clara, CA, USA). Chromatography was performed on a UPLC $\mathrm{C}_{18}$ analytical column $\left(2.1 \times 5 \mathrm{~mm}\right.$, I.D. $1.7 \mu \mathrm{m}$, ACQUITY UPLC ${ }^{\circledR}$ BEH, Waters, Milford, MA, USA). The mobile phase used for the elution of the column consisted of $0.1 \%(\mathrm{v} / \mathrm{v})$ formic acid in water (solvent $\mathrm{A}$ ) and $0.1 \%(\mathrm{v} / \mathrm{v})$ formic acid in acetonitrile (solvent $B$ ). A gradient elution was performed as follows: $0-15 \mathrm{~min}, 10-45 \% \mathrm{~B} ; 15-23 \mathrm{~min}, 45-70 \% \mathrm{~B}$; $23-25 \mathrm{~min}, 70-100 \% \mathrm{~B}$. The flow rate was set at $0.4 \mathrm{~mL} /$ min with an injection volume of $2 \mu \mathrm{L}$, whilst the column temperature was maintained at $40{ }^{\circ} \mathrm{C}$.

Detection by ESI-QTOF-MS/MS was performed in positive ion mode. The source parameters were set as follows: gas temperature, $300{ }^{\circ} \mathrm{C}$; gas flow, $8.0 \mathrm{~L} / \mathrm{min}$; nebulizer pressure, $45 \mathrm{psi}$; sheath gas temperature, $350{ }^{\circ} \mathrm{C}$; sheath gas flow, $8.0 \mathrm{~L} / \mathrm{min}$. The scan source parameters were set as follows: VCap, 3500; nozzle voltage, $500 \mathrm{~V}$; fragmentation voltage, $120 \mathrm{~V}$. Reference masses were used at $m / z 121.0508$ (purine) and 922.0097 (hexakisphosphazine). Automatic MS/MS was performed using a fixed collision energy of $15 \mathrm{eV}$.

\section{Qualitative analysis}

Data were analyzed using the Agilent MassHunter Workstation Software-Qualitative Analysis software (version B.06.00, build 6.0.633.0, Agilent Technologies Inc., 2012) with the following settings: extraction restrict retention time, $2-25 \mathrm{~min}$; peak height, $\geq 100$ counts; charge state, 1 ; peck spacing tolerance, $0.0025 \mathrm{~m} / z$ plus 
$7.0 \mathrm{ppm}$; compound relative height, $\geq 2.5 \%$; absolute height, $\geq 2000$ counts; elements of $\mathrm{C}, \mathrm{H}, \mathrm{O}$ from 3 to 60,0 to 120 and 0 to 30 , respectively, for generating the formulae.

\section{Statistical analysis}

Statistical analyses and class prediction of the UPLCMS/MS results were carried out using the Agilent MassHunter Mass Profiler Professional Software (version B.02.02, Agilent Technologies Inc., 2011). Data were initially exported from the Mass Hunter Workstation before being imported into the Mass Profiler Professional Software with the following settings: abundance filtering with minimum absolute abundance, 5000 counts; all charge states permitted; retention time (RT) correction with a maximum of $0.5 \%$ plus $0.5 \mathrm{~min}$; mass window $5.0 \mathrm{ppm}$ plus $2.0 \mathrm{mDa}$; compound alignment with RT window $0.1 \%$ plus $0.15 \mathrm{~min}$. One-way analysis of variance (ANOVA) was used with a $P$ value of 0.05 to further filter these data. A 3D principal component analysis (PCA) was conducted on all of the samples. A hierarchical cluster analysis (HCA) was also conducted with the following settings: cluster on conditions, Euclidean as the distance metric, centroid as the linkage rule. An automated sample class prediction model was built based on the UPLCMS/MS results of the reference plants with the following settings: Naive Bayes as the class prediction algorithm; validation type, $\mathrm{N}$-fold; number of folds, 3 ; number of repeats, 10.

SIMCA (version 13.0.3.0, Umetrics AB 2013) was used to analyze selected peaks. The peak volumes were manually imported as X-variables, with the sample number as a primary ID, peak number as a secondary ID and sample speciation as a class ID. The model type was set using orthogonal partial least squared discriminant analysis (OPLS-DA), where the significant components were calculated using an "Autofit" function.

\section{Results and discussion}

\section{Chromatographic profiling of the reference plants}

The UPLC-ESI-QTOF-MS/MS chromatograms revealed the chemical profiles of the three different Isodon species, and typical base peak chromatograms (BPC) of these three species are shown in Fig. 3. The chemical profiles of IL and ILG were similar, whereas the chemical profile of IS was considerably different. By comparing the samples of the different batches of IS, IL and IL, we were able to select 11,17 and 13 common peaks for each species, respectively. One common peak (peak 5) was found in all three species. Thirteen common peaks were found in the chromatograms of IL and ILC, namely peaks $1,2,5,13$, $14,19,21,22,23,24,25,26$ and 27. The main difference between IL and ILG was that peaks 4, 16, 17 and 20 only appeared in IL. Moreover, peak 13 was absent or only detected in small quantities in ILG, but was distinct in IL; peak 14 was absent or only detected in small quantities in IL, but was distinct in ILG; peak 22 was present in much higher quantities in IL than ILG; and peak 27 was detected in higher quantities than peak 26 in IL, and vice versa in ILG. However, 10 specific peaks (peaks 3, 6, 7, 8, $9,10,11,12,15$ and 18 ) were only detected in the chromatogram of IS.

All of the raw UPLC-MS data for the reference plants were examined using the Agilent MassHunter Mass Profiler Professional Software to further validate the differences between the three Isodon species. PCA was conducted, which exposed the variance between the samples by converting the numerous variables into principal components. The UPLC-MS data for the plants were imported for the calculation. Following the reduction of these data by statistical means, including ANOVA and the use of a frequency filter, three principal components were calculated. The PCA plot with principal components 1 (41.20\%), $2(22.26 \%)$ and $3(7.42 \%)$ is shown in Fig. 4. The samples were distinctively clustered in three groups, which were correlated with the three species. The UPLC-MS features of the three species were different and these data could be used to distinguish between the three species. HCA was also used to interpret the relationships between the three different species. By comparing the detected $m / z$ signals and signal intensities of the UPLCMS data, samples were organized into clusters based on the similarity in their MS profiles. Figure 5 shows the dendrogram of hierarchical clustering, where the samples have been grouped into three branches correlating to the three species. These data highlighted the closer 


\section{a}

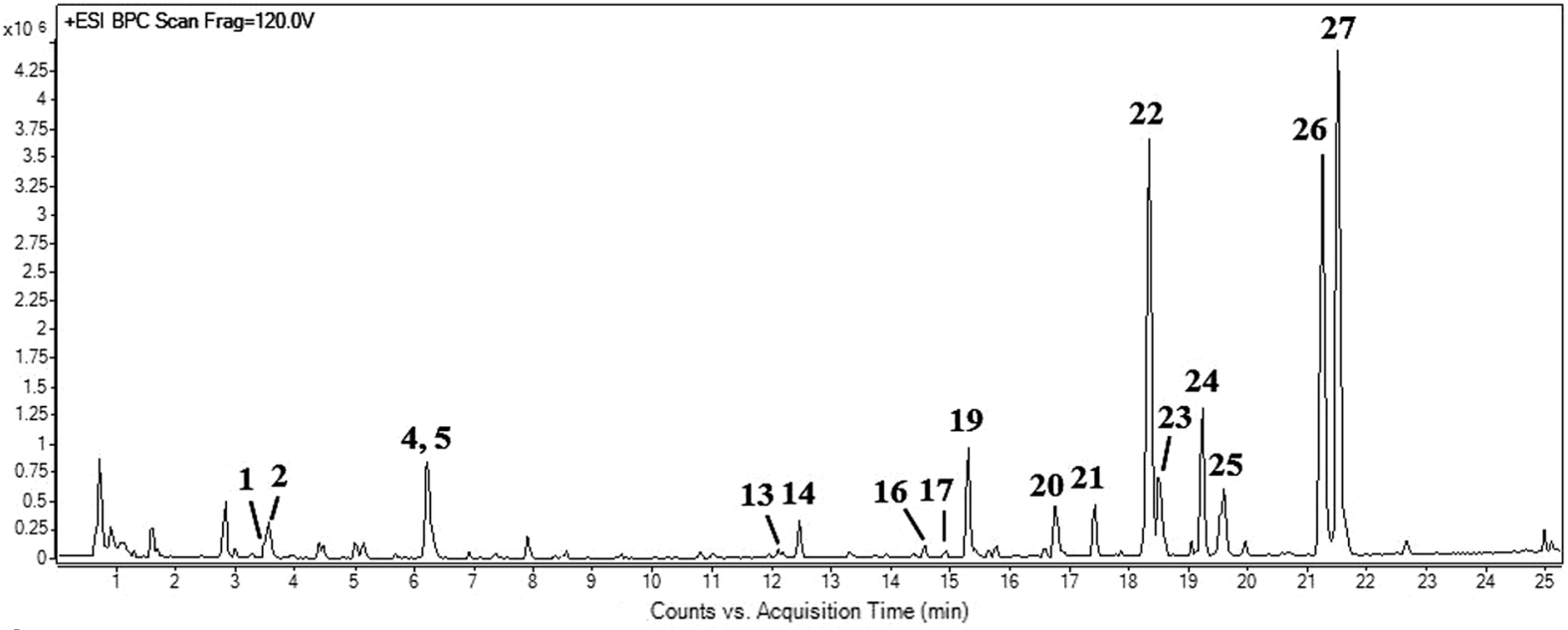

b

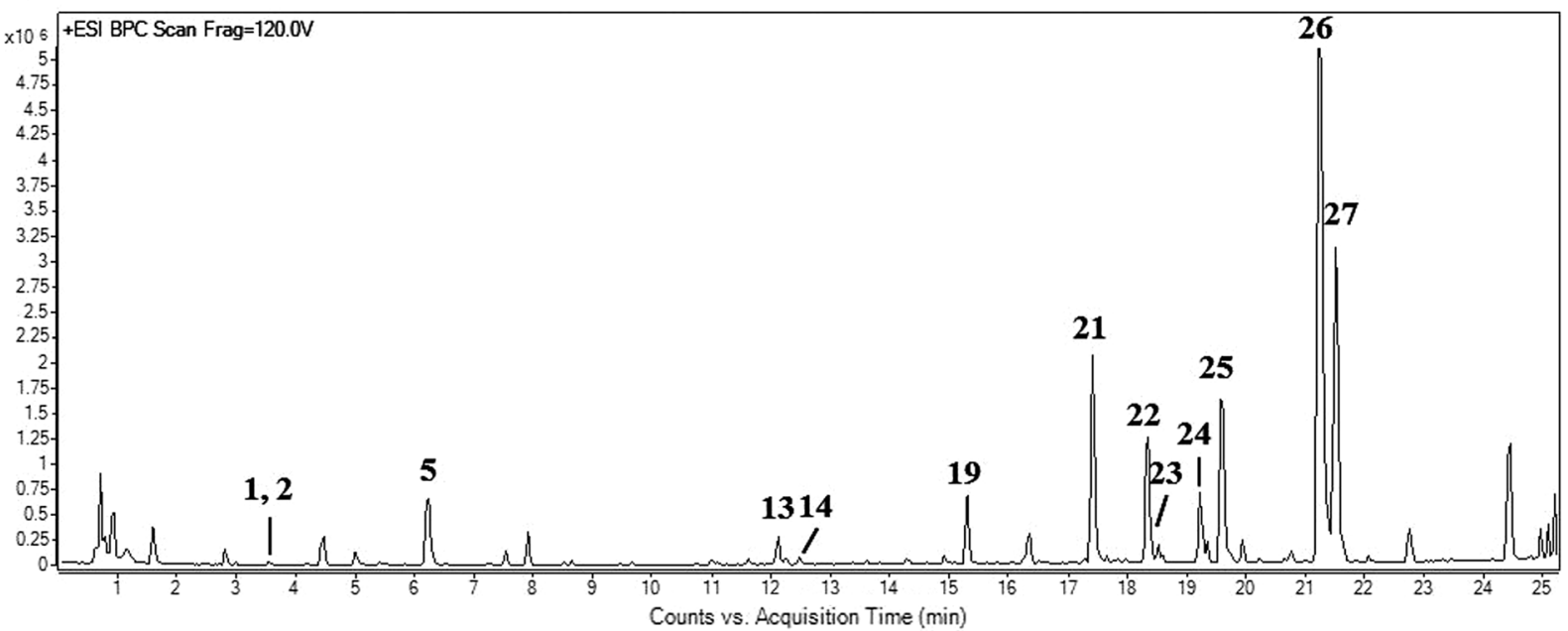

C

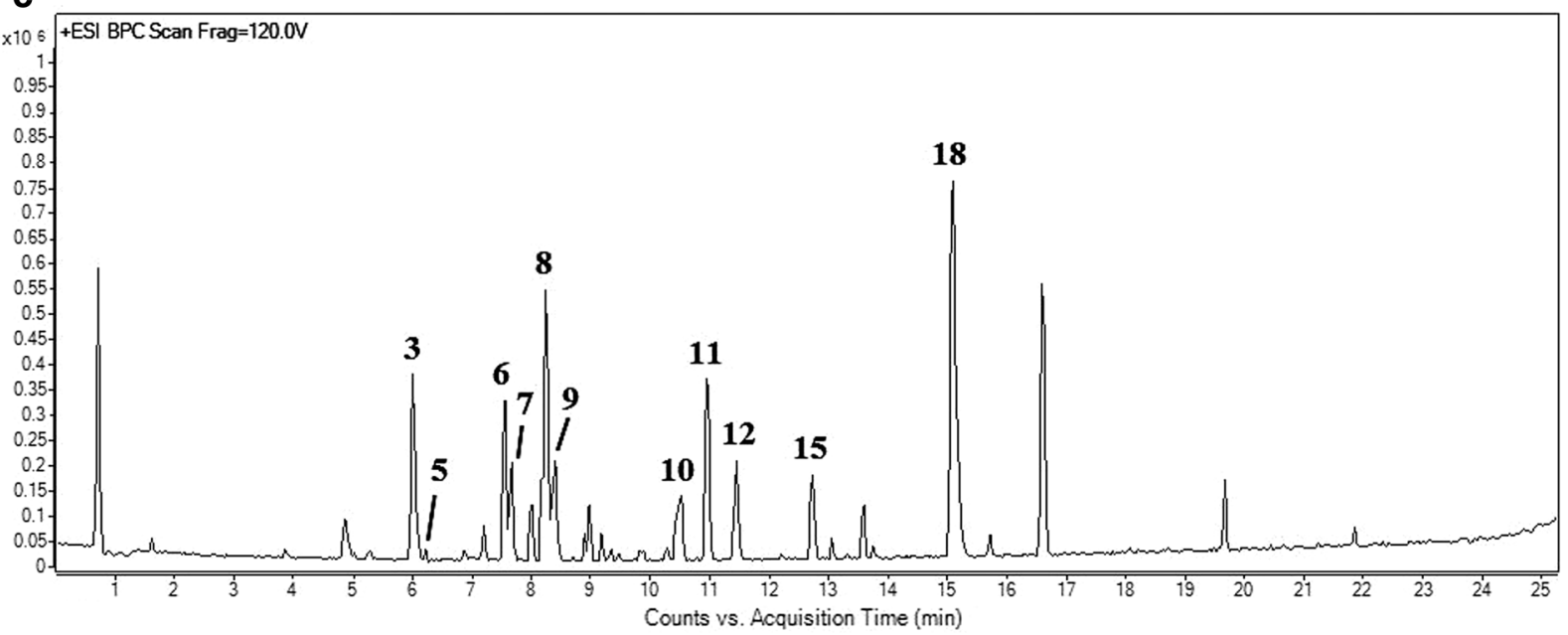

Fig. 3 Typical BPC chromatograms of a I. lophanthoides, b I. lophanthoides var. graciliflorus and c I. serra 


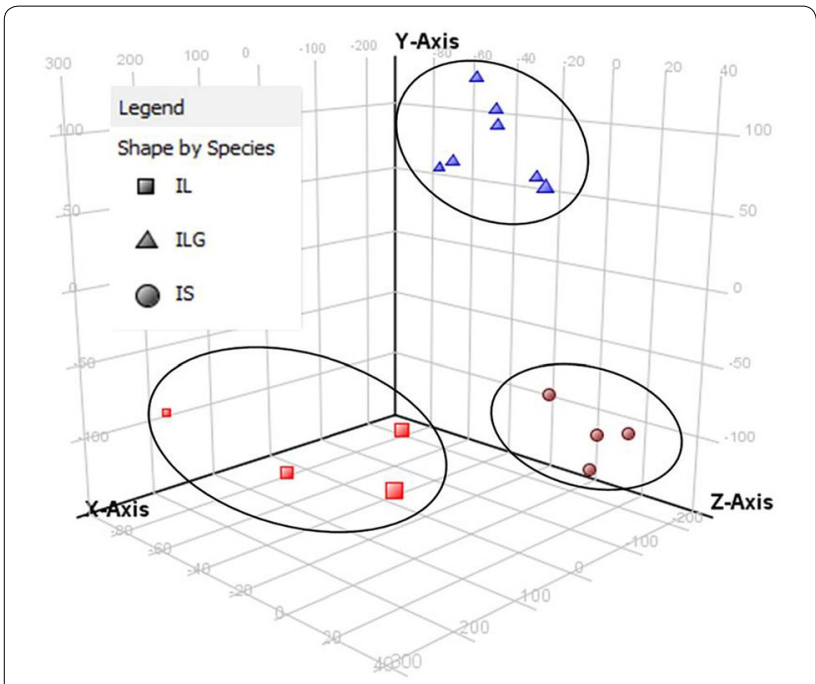

Fig. 4 3D PCA plot of the samples

relationship between IL and ILG compared with IS. The HCA results therefore supported those of the PCA, and revealed the phylogenetic relationships between the three Isodon species.

Twenty-seven different peaks were selected from the chromatograms of the three different species to allow for their differentiation. Furthermore, the ability of these peaks to distinguish between the different species was verified by OPLS-DA. A 3D OPLS-DA is shown in Fig. 6 , where the samples were separated into three groups by species. The consistency observed between these results and those obtained using the MS data highlighted the representative nature of the selected peaks.

Peak assignments and the interpretation of MS/MS spectra Peak assignments for the three species were conducted according to the references, standard compounds and possible fragmentation pathways. The details of the peaks identified from the three Isodon species are summarized in Table 3, and the structures of the identified compounds are shown in Fig. 7.

Peak 5 was common to all three species. The dominant mass ion for peak 5 had an $m / z$ value of 163.0392 , which was consistent with the MS data of rosmarinic acid resulted from the cleavage of its ester bond, weak $\mathrm{m} / z$ 383.0724 $[\mathrm{M}+\mathrm{Na}]^{+}$and $m / z$ 343.0814 $\left[\mathrm{M}+\mathrm{H}-\mathrm{H}_{2} \mathrm{O}\right]^{+}$ ions also found, which provided evidence that this peak could be attributed to rosmarinic acid $[12,14]$.

Diterpenoids are the major active compounds in Isodon species [15]. More than 500 diterpenoids have been isolated from plants belonging to this genus and a number of them have been reported to exhibit strong antibacterial, anti-inflammatory and anticancer activities [15]. Six ent-kaurane type diterpenoids were identified from IS in this study, including lasiodonin (peak 3), oridonin (peak 7), ponicidin (peak 8), lasiokaurin (peak 10), shikokianin (peak 13) and shikokianidin (peak 18) [11, 16]. Their chemical structures are shown in Fig. 7. The loss of $\mathrm{H}_{2} \mathrm{O}(-18 \mathrm{Da})$ from a hydroxyl group, $\mathrm{AcOH}(-60 \mathrm{Da})$ or $\mathrm{CH}_{2}=\mathrm{CO}(-42 \mathrm{Da})$ from an acetate group, $\mathrm{CO}(-28 \mathrm{Da})$ from the $\mathrm{D}$ ring and $\mathrm{CH}_{2} \mathrm{O}(-30 \mathrm{Da})$ from the $\mathrm{C}-20$ oxygenated $\mathrm{B}$ ring were identified as the dominant fragmentation processes in the tandem MS/MS spectra of these compounds.

Abietane and tricyclic diterpenoids have been reported to be the major diterpenoids found in IL and ILG. Diterpenoids of this type have only ever been found in a few Isodon species, including I. grandifolia and I. forrestii [17]. Information pertaining to diterpenoids of this type is therefore limited. Based on the fragmentation pattern of salvialeriafone, which is a diterpenoid found in the genus Salvia with a similar skeletal structure to those found in IL and ILG, the proposed fragmentation of the diterpenoids was the loss of $\mathrm{CO}(-28 \mathrm{Da})$ and $\mathrm{H}_{2} \mathrm{O}$ $(-18 \mathrm{Da})$ from their $\mathrm{C}$ ring after the removal of their side chains or other functional groups [18]. Although more than thirty diterpenoids were isolated from IL, ILG, $I$. lophanthoides var. gerardianus and I. lophanthoides var. micranthus [6, 8-10, 19], none of them showed identical molecular weight and possible fragmentation pathways to the peaks in this study. However, the calculated formulae and MS/MS fragments $-\mathrm{C}_{3} \mathrm{H}_{4}(-40 \mathrm{Da}),-\mathrm{C}_{3} \mathrm{H}_{6}(-42 \mathrm{Da})$ and $-\mathrm{C}_{5} \mathrm{H}_{8}(-68 \mathrm{Da})$ (removal of side chain from $\mathrm{C}$ ring) indicated that most of the peaks could be attributed to abietane-type diterpenoids. Furthermore, two of the peaks in the chromatograms for IL and ILG were identified as glycosides schaftoside (peak 1) and isoschaftoside 


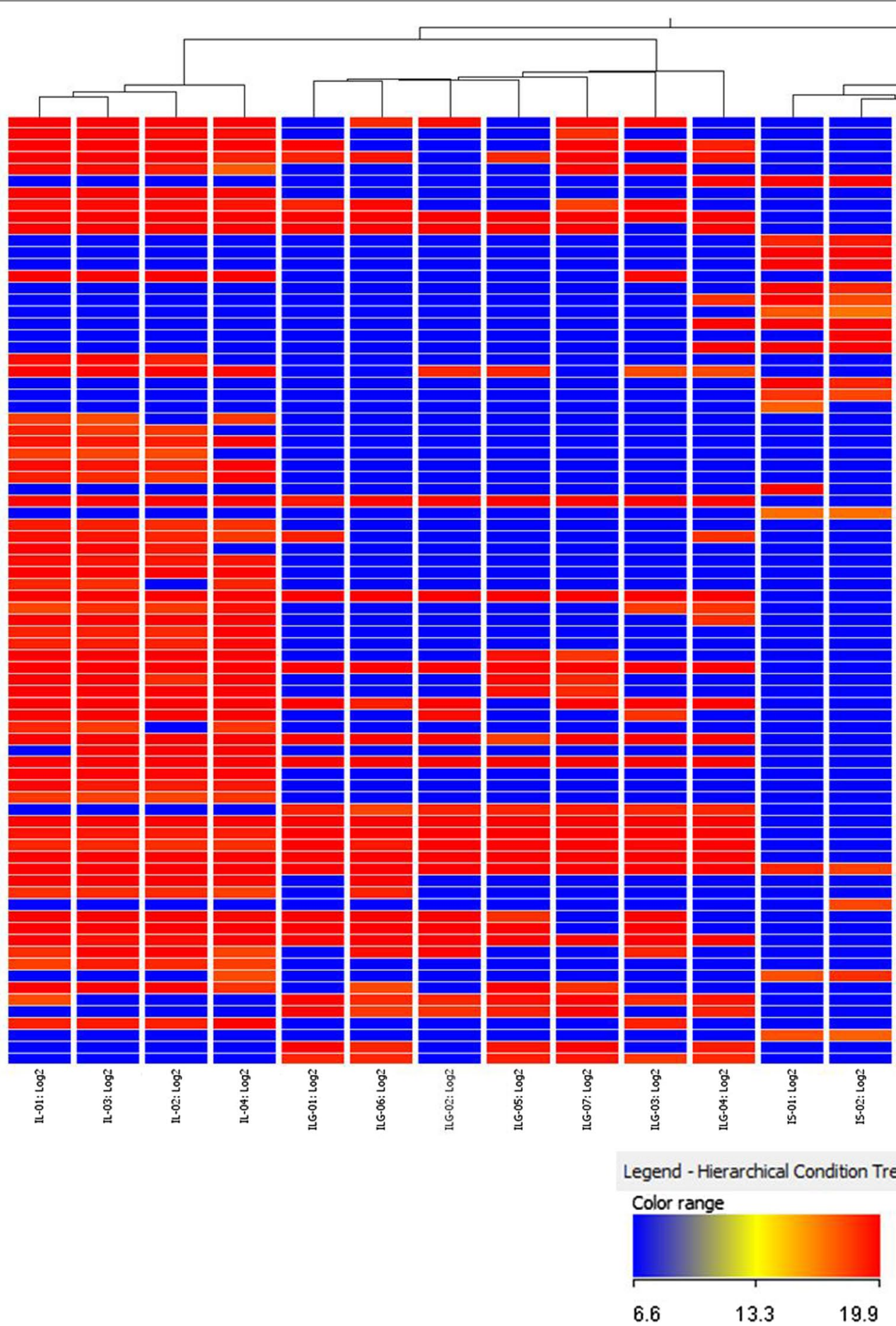

Fig. 5 Hierarchical cluster analysis heat map for the association of compounds detected in samples. Each line represents an $\mathrm{m} / \mathrm{z}$ signal detected in MS, whereas the color indicates the $\log _{2}$-transformed $m / z$ signal intensities 


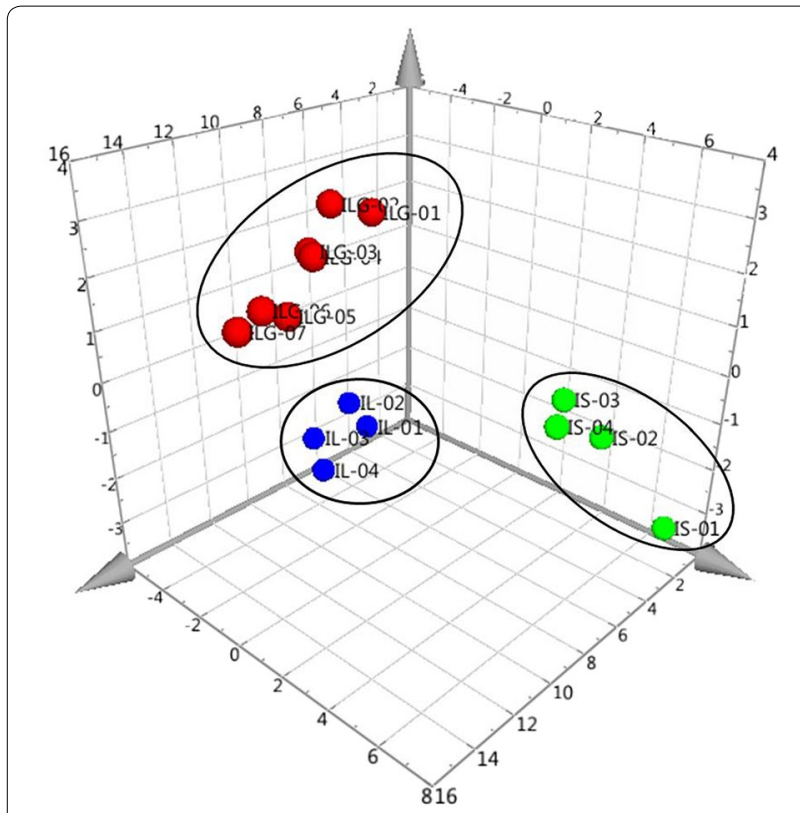

Fig. 6 The OPLS-DA plot of samples using the selected peaks

(peak 2) based on comparisons with standards and references $[13,20]$.

\section{Identification of commercial samples}

Based on the UPLC-MS data generated using the reference plants, we proceeded to investigate the identification of the 27 batches of commercial Xihuangcao. Fundamental morphologic and microscopic identification of Xihuangcao and several related species were done in our previous study. In terms of the morphological features of the 27 samples evaluated in this study, eight of the samples (samples 03, 04, 06, 09, 11, 14, 15, and 20) obviously did not contain plants belonging to the genus Isodon, which have white powders on their surface and leaves 1- or 2-pinnate (Fig. 2d). The microscopic features of these samples were also different from those of the Isodon species according to our preliminary studies. These samples were therefore identified as Ampelopsis grossedentata (Hand.-Mazz.), which is a common adulterant of Xihuangcao [21]. Preliminary analysis of A. grossedentata by UPLC-MS showed its chemical composition differed considerably from those of the Isodon species (Additional file 1: Figure S1), hence the UPLC-MS data for these eight batches were excluded from the automated sample class prediction.

A prediction model was initially built using the UPLC-MS data generated form the 15 reference plants. Significant signals were identified via a series of statistical calculations and filtration processes, followed by a statistical validation step, which showed the accuracy of the prediction for each class. The model was finally visualized by PCA. In this model, naive Bayesian classifier, which is a multi-class parameter-based statistical classifier, was used. The UPLC-MS data of the remaining 19 commercial samples were imported and classified using the prediction model. Twelve samples were predicted as ILG (samples 01, 02, 08, 13, 16, 19, 21, 22, 23, 24, 25 and 27), whereas six samples were predicted as IS (samples $05,07,10,12,18$ and 26) with a high confidence measure of 1.000 in both cases. The only major exception was sample 17, which was predicted to be IS with a low confidence measure of 0.692 . A representative prediction report is shown in Fig. 8. The UPLC-MS data for this sample was converted into three principal components and compared with those of the model. Manual identification of the samples using the 27 characteristic peaks was also conducted to further confirm the result. All of these results were consistent with those of the automated prediction, except for sample 17 , which showed a low confidence measure. This sample could not be identified as one of the three Isodon species.

According to the species identification results, ILG was the major source of Xihuangcao, whereas IS was found in approximately one-quarter of the samples (22\%) and IL was not used at all. Xihuangcao is mainly sold as a special local product in the markets for the preparation of soup or herbal tea. IS has a very bitter taste, whereas IL and ILG are only slightly bitter, which could explain why IS 


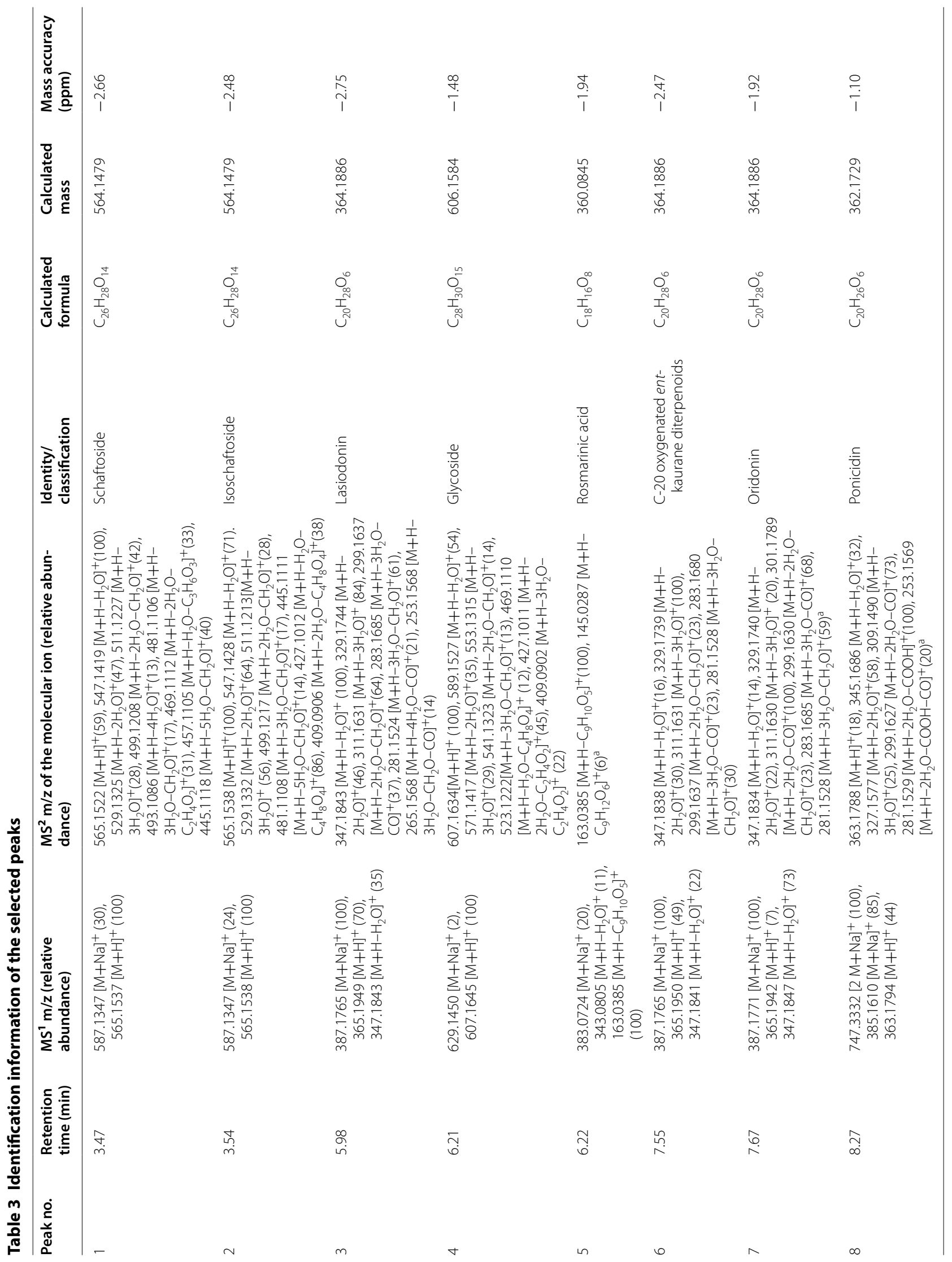




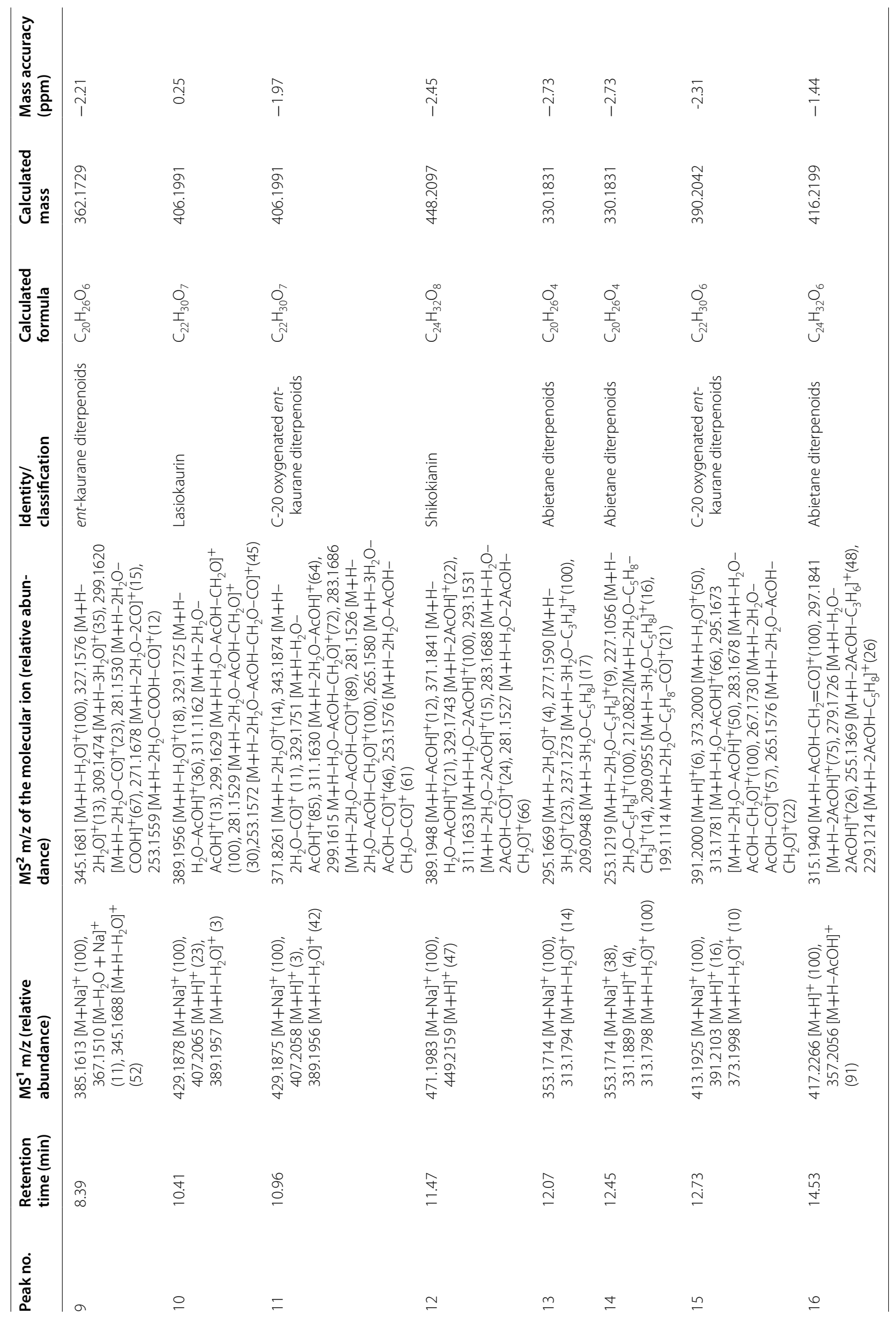




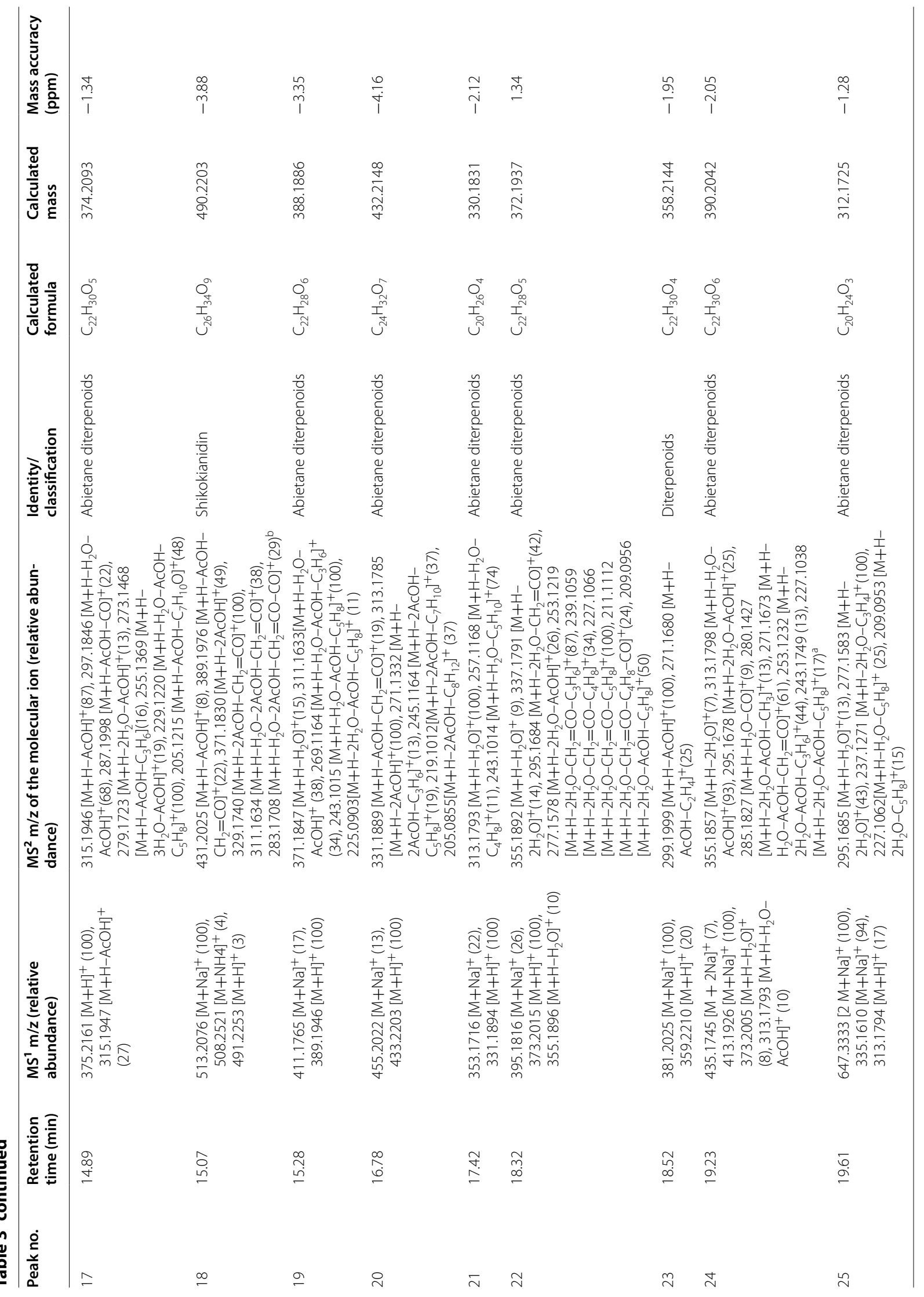




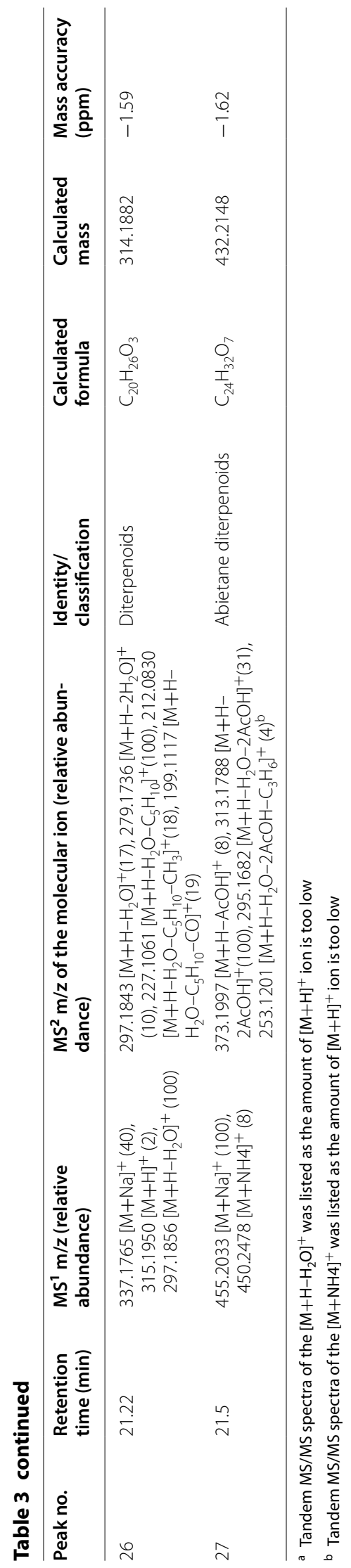


<smiles>O=c1cc(-c2ccc(O)cc2)oc2c(C3OCC(O)C(O)C3O)c(O)c(C3OC(CO)C(O)C(O)C3O)c(O)c12</smiles>

a<smiles>O=c1cc(-c2ccc(O)cc2)oc2c(C3OC(CO)C(O)C(O)C3O)c(O)c(C3OCC(O)C(O)C3O)c(O)c12</smiles>

b<smiles>O=C(/C=C/c1ccc(O)c(O)c1)OC(Cc1ccc(O)c(O)c1)C(=O)O</smiles>

C<smiles>C=C1C(=O)C23CC1CC(O)C1C2(O)C(O)C(O)C2(C)OC13C(C)(C)CCC2O</smiles>

d<smiles>C=C1C(O)C2(C(=O)O)C1CCC1C3C(O)C2(O)OC1(C(=O)O)C(OC(=O)O)CCC3(C)C</smiles>

g<smiles>C=C1C2CCC3C45C=CCC(C)(C)C4C(O)C3(C5)OC1(O)C2C(=O)O</smiles>

e<smiles></smiles>

h

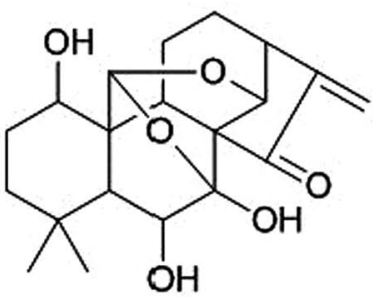

f

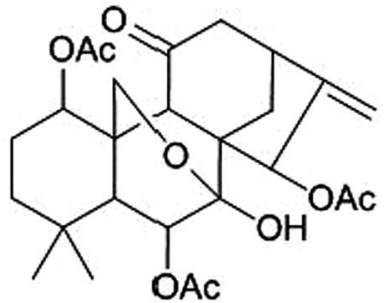

i

Fig. 7 Chemical structures of the identified compounds. a Schaftoside; b Isoschaftoside; $\mathbf{c}$ Rosmarinic acid; $\mathbf{d}$ Lasiodonin; $\mathbf{e}$ Oridonin; $\mathbf{f}$ Ponicidin; $\mathbf{g}$ Lasiokaurin; h Shikokianin; i Shikokianidin

was found in small quantities in the Xihuangcao samples in this market investigation. IL is an annual or short-lived perennial herb, whereas its variety ILG is an undershrub capable of reaching more than $1 \mathrm{~m}$ in height [3]. ILG is therefore more desirable for cultivation in terms of its production yield. The large proportion of adulterant (44\%) found in this study also raises concerns about the quality of the Xihuangcao being sold in retail markets. 

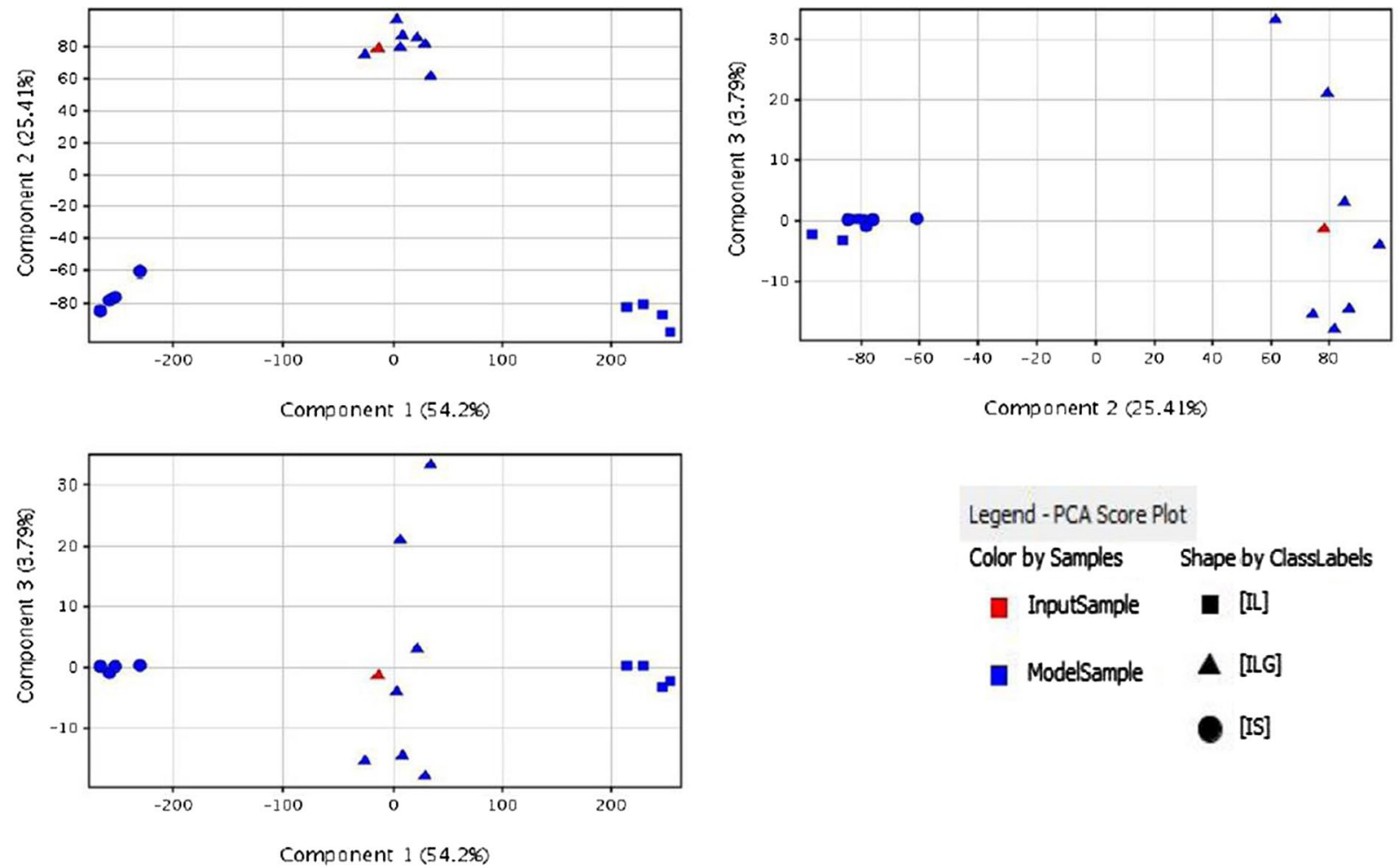

Fig. 8 Prediction report of sample 02

\section{Conclusion}

UPLC-ESI-QTOF-MS and subsequent chemometrics analysis were demonstrated effective to differentiate among the three different species of plants used as Xihuangcao.

\section{Additional file}

Additional file 1: Figure S1. The BPC chromatograms of Ampelopsis grossedentata.

\section{Abbreviations}

BPC: base peak chromatogram; HCA: hierarchical cluster analysis; IL: Isodon lophanthoides; ILG: Isodon lophanthoides var. graciliflorus; IS: Isodon serra; MS: mass spectrometry; ANOVA: one-way analysis of variance; OPLS-DA: orthogonal partial least squared discriminant analysis; PCA: principal component analysis; UPLC: ultra-performance liquid chromatography; UPLC-ESI-QTOF-MS: ultra-performance liquid chromatography coupled with electrospray ionization quadrupole time-of-flight mass spectrometry.

\section{Authors' contributions}

HBC and ZZZ conceived the study. LLW and ZTL designed the study. LLW, $Z T L$ collected the plant materials and samples. LLW conducted the sample preparation, UPLC analysis, and analyzed the data. LLW, ZTL wrote the manuscript. ZTL revised the manuscript. All authors read and approved the final manuscript.

\section{Author details}

${ }^{1}$ School of Chinese Medicine, Hong Kong Baptist University, Kowloon, Hong Kong Special Administrative Region, People's Republic of China. ${ }^{2}$ Research Center for Pharmacognosy, Institute of Chinese Materia Medica, Academy of Chinese Medical Sciences, Beijing, People's Republic of China.

\section{Acknowledgements}

We would like to thank Ms. Qiaohua Deng and Mr. Weiliang Zhang form Hutchison Whampoa Guangzhou Baiyunshan Chinese Medicine Company Limited for the help in the collection of reference plants, also Mr. Alan Ho from the School of Chinese Medicine, Hong Kong Baptist University for his kind technical support.

\section{Competing interests}

The authors declare that they have no competing interests.

Received: 13 September 2016 Accepted: 17 November 2016

Published online: 15 December 2016

\section{References}

1. Wu JF. Literature review of Xihuangcao. Shi Zhen Guo Yi Guo Yao. 2003;14(8):498-500.

2. Guangdong Food and Drug Administration. Guangdong Chinese Materia Medica Standards, vol. 2. Guangdong: Guangdong Science \& Technology Press: 2011. p. 347-53.

3. Suddee S, Paton AJ, Parnell JAN. A taxonomic revision of tribe Ocimeae Dumorts. (Lamiaceae) in continental South East Asia. Kew Bull. 2004:59(3):379-414 
4. Fu L, Deng QH, Ceng FY, Yr YS. Research of the name of Chinese medicine Xihuangcao. Zhong Yao Cai. 2012;35(3):493-5.

5. Chen JN, Jiang DX, Zhao AG, Lai XP. Microscopic identification of medicinal material of Herba Rabdosiae Serrae. Guang Zhou Zhong Yi Yao Da Xue Xue Bao. 2006;23:52-5.

6. Zhang Q, Pan CM. The comparative studies on the morphology of I. serra, I. Iophanthoides, I. lophanthoides var. geradiana and I. lophanthoides var. graciliflorus. Pharm Today. 2012;22(1):21-4.

7. Liang YG, Xie HH, Wu P, Jiang YM, Wei XY. Podocarpane, isopimarane, and abietane diterpenoids from Isodon lophanthoides var. graciliflorus. Food Chem. 2013:136:1177-82.

8. Yang LB, Li L, Huang SX, Pu JX, Zhao Y, Ma YB, Chen JJ, Leung CH, Tao ZM, Sun HD. Anti-hepatitis B virus and cytotoxic diterpenoids from Isodonlophanthoides var. gerardianus. Chem Pharm Bull (Tokyo). 2011;59(9):1102-5.

9. Zhao AH, Li SH, Li YW, Huan QB, Zhao QS, Lin ZW, Sun HD. Two New Abietane Quinones from Isodon lophanthoides var micranthus. Chin Chem Lett. 2003:14(6):591-3.

10. Zhou W, Xie H, Wu P, Wei X. Abietane diterpenoids from Isodon lophanthoides var. graciliflorus and their cytotoxicity. Food Chem. 2013;136:1110-6.

11. Liu PW, Du TF, Zhang XW, Sheng XN, Shi XW, Zhai CC, Zhu H, Wang N, Zhang LT. Rapid analysis of 27 components of Isodon Serra by LC-ESI-MSMS. Chromatographia. 2010;72:265-73.

12. Flora of China: Taxonld 210000832-Isodon serra. Beijing: Flora of China Editorial Board. 2013. http://foc.eflora.cn/content. aspx?Taxonld=210000832. Accessed 12 Jun 2014.
13. Kuang $\mathrm{YH}$, Liang $\mathrm{S}$, Yao $\mathrm{XH}$, Huang L, Lin Q. Determination of isoschaftoside, schaftoside and romarinic acid in Isodon lophanthoides var. graciliflora by HPLC. Zhong Cheng Yao. 2013;35(3):570-3.

14. MassBank Record: PR040220—Rosmarinic acid. Tokyo: MassBank Project. 2014. http://www.massbank.jp/jsp/Dispatcher.jsp?type=disp\&id=PR040 220\&site $=1$. Accessed 12 Jun 2014.

15. Sun HD, Huang SX, Han QB. Diterpenoids from Isodon species and their biological activities. Nat Prod Rep. 2006;23:673-98.

16. Sun HD, Xu YL, Jiang B. Diterpenoids from Isodon species. Beijing: Science Publishing House; 2001. p. 245.

17. Zhang L, Zhou GX, Li Q, Dai Y, Ye WC, Yao XS. Chemical constituents of Isodon lophanthoides (Buch. -Ham. ex D. Don) Hara var. gerandianus (Benth.) Hara. Shen Yang Yao Ke Da Xue Xue Bao. 2006;23(12):768-70,

18. Musharraf SG, Goher M, Hussain A, Choudhary ML. Electrospray tandem mass spectrometric analysis of a dimeric conjugate, salvialeriafone and related compounds. Chem Cent J. 2012;6:120.

19. Sun HD, Xu YL, Jiang B. Diterpenoids from Isodon species. Beijing: Science Publishing House; 2001. p. 119.

20. Colombo R, Yariwake JH, McCullaghb M. Study of C- and O-glycosylflavones in sugarcane extracts using liquid chromatography —exact mass measurement mass spectrometry. J Braz Chem Soc. 2008;19(3):483-90.

21. Deng QH. Study on distribution and development of Herbra Isodonis Lophanthoidis. Jin Ri Yao Xue. 2009;19(9):21-5.

\section{Submit your next manuscript to BioMed Central and we will help you at every step:}

- We accept pre-submission inquiries

- Our selector tool helps you to find the most relevant journal

- We provide round the clock customer support

- Convenient online submission

- Thorough peer review

- Inclusion in PubMed and all major indexing services

- Maximum visibility for your research

Submit your manuscript at www.biomedcentral com/submit 